\title{
Adaptive Technique for CI/MC-CDMA System using Combined Strategy of Genetic Algorithms and Neural Network
}

\author{
Santi P. Maity \\ Department of Information Technology, \\ Bengal Engineering and Science University, Shibpur \\ Howrah, 711 103, West Bengal (India) \\ Tel: +91-9830023316 E-mail: santipmaity@it.becs.ac.in
}

Sumanta Hati

Dept. of Information Technology, Bengal Engineering and Science University

Shibpur, Howrah, 711 103, West Bengal (India)

Tel: +91-9883112079 E-mail: sumanta.hati@ rediffmail.com

Received: January 31, 2012

DOI: $10.5296 /$ npa.v4i1.1330
Accepted: May 16, $2010 \quad$ Published: May 30, 2010

URL: http://dx.doi.org/10.5296/npa.v4i1.1330

\begin{abstract}
Multicarrier Code Division Multiple Access (MC-CDMA) is one of the most promising techniques for high bit rate and high user capacity transmission in future broadband mobile services. The use of carrier interferometry (CI) codes further improves this user capacity relative to the conventional spreading codes. Genetic Algorithms (GA) may be used to find the optimum transmitted powers that maximize the channel transmission capacity as well as to reduce bit error rate (BER) values. On the other hand, neural networks (NN) are trained to optimize the weight factors in minimum mean square error combining receiver (MMSEC) via back propagation type algorithm. Optimum values of weight factors give stable decision variables that lead to improved receiver performance without having the knowledge of channel state information (CSI) and transmit signal powers. Decision variables are then used for realization of an efficient block parallel interference cancellation (BPIC) as multiuser detection (MUD). Simulation results show that BER performance using GA-NN is better than any other existing works.
\end{abstract}


Keywords: MC-CDMA, Carrier Interferometry Code, Power Allocation, PIC, Neural Network, Genetic Algorithms.

\section{Introduction}

On growing demand of data intensive applications such as interactive and multimedia services, the need of reliable and high rate data transmission over a wireless mobile channel becomes essential. However, high data rate transmission increases the intersymbol interference (ISI) caused by the time dispersive nature of the radio mobile channel. In code division multiple access (CDMA), multiple number of users share the same channel bandwidth at the same time through the use of (near) orthogonal spreading codes. So it has potential to provide higher user capacity compared to its other close competitors such as time division multiple access (TDMA) and frequency division multiple access (FDMA). Multicarrier code division multiple access (MC-CDMA), which is a combination of orthogonal frequency division multiplexing (OFDM) and CDMA, provides a flexible multipath propagation. OFDM, (hence MC-CDMA also) reduces ISI by introducing a guard interval which is a cyclic extension of any multicarrier signal.

Development of spreading codes had drawn a great interest since inception of CDMA with an objective to increase in user capacity with low multiple access interference (MAI). A thorough analysis and comparison of existing MC-CDMA codes that include Hadamard-Walsh, Gold, orthogonal Gold, Zadoff Chu sequences is presented in [1]. Wavelet and subband theories are also used to develop a multivalued set of orthogonal codes [2]. The widely used Hadamard-Walsh codes support either ' $\mathrm{N}$ ' users orthogonally or more than $\mathrm{N}$ users pseudo-orthogonally at the cost of degraded performance. Recently, the concept of interferometry is exploited to develop set of code patterns with carefully chosen phase offsets that ensures a periodic main lobe in time domain and side lobe activity at intermediate times. The usage of carrier interferometry (CI) codes in MC-CDMA system supports $\mathrm{N}$ users orthogonally and additional N-1 users pseudo-orthogonally. Moreover, there is no restriction on the length $\mathrm{N}$ of the $\mathrm{CI}$ codes (i.e., $\mathrm{N} \in \mathrm{I}$ ), unlike Hadamard Walsh codes where $\mathrm{N}$ is limited to $2^{\mathrm{n}}$ or $2^{\mathrm{n}} \pm 1(\mathrm{n} \in \mathrm{I})[3]$.

According to Shannon channel capacity theorem, increase in signal power increases signal-to-noise ratio (SNR) for a given noise power. This in turn improves the system's channel capacity (c) i.e. data transmission rate for single user communication. However, in the presence of multiple users, if all users try to increase their data rates by increasing their transmit power, the users interfere. As a matter of fact both the signal and interference power increase. Consequently, the signal-to-interference plus noise power- ratio (SINR) and therefore the users' rates saturate at a constant value [4]. In other words, high SNR values of other users act as strong interfering effect to the nearby users in multiuser environment transmitting in the same set of subcarriers. This suggests that optimum power allocation is essential in order to increase channel capacity and to reduce BER values. This is often done by exploiting the channel state information (CSI) that is fed back from transmitter to receiver $[5]$. 
In radio mobile communication, minimum mean square error combining (MMSEC) receiver has shown to provide the best performance in frequency selective fading channel using the CSI. However, CSI estimation may not be accurate always and may also be outdated due to feedback delay. To alleviate this problem, calculation of proper weight factors in MMSEC correlator becomes important so that receiver performance can be improved without having knowledge of transmission power and CSI. Weight factors calculation may be done through learning/training. Hence, neural network (NN), due to its inherent learning and adaptive capability, may be used. Furthermore, multiuser detection (MUD) in CDMA can be used efficiently to improve receiver performance exploiting the cancelation of MAI. Among various MAI cancelation strategies, successive interference cancelation (SIC), parallel interference cancelation (PIC) and its various variants like partial PIC (PPIC), block PIC (BPIC) have been proposed and related literature is quite rich.

In this paper, we have developed an efficient power allocation scheme in CI/MC-CDMA system using combined Genetic algorithms (GA) and NN. GA is used here to optimize the transmitted power for each user in respective subcarrier in order to reduce interfering effect with a hope to increase channel capacity. On the other hand, $\mathrm{NN}$ is used here to calculate the subcarrier weight factors in MMSEC of different users under frequency selective Rayleigh fading channel. $\mathrm{NN}$ is trained to optimize the weight factors via back propagation type algorithm. Experiment is carried out through simulation on frequency selective Rayleigh fading channel. Results show that channel capacity and bit error rate (BER) performance obtained for the adaptive system is significantly better than the non-power adaptive system.

The rest of the paper is organized as follows: Section 2 presents review of the prior works, limitations and scope of the present work. Section 3 describes system model for synchronous CI/MC-CDMA uplink system. Section 4 represents proposed GA based power allocation algorithm, while Section 5 presents NN assisted MMSEC receiver with BPIC model. Section 6 presents performance evaluation and with discussion. Finally conclusions are drawn in Section 7 along with the scope of future work.

\section{Review of Prior Works, limitations and scope of the present work}

Channel capacity improvement by adaptive power allocation in multicarrier system is an important research topic and several solutions are reported in literature. In [6], Shen et al propose an optimal power allocation algorithm in their OFDM system that maximizes the sum capacity and at the same time each user maintains a required data rate. Luo et al [7] studied the power allocation problem in decode and forward cooperative relaying system. The algorithm proposed an equal power allocation with a channel selection algorithm based on minimizing the outage probability. An algorithm for optimal transmitted power control is proposed by Zhang et al in [8]. They consider controlled transmit power for a two-band system as a linear function of the power attenuation difference (between the two bands) for a large range of these attenuation differences. 
Multiuser detection in CDMA has gained wide popularity over the last decade and literature is quite rich. The optimum multiuser detector proposed in [9] achieves significant performance improvement relative to single user receivers but the computational complexity increases exponentially with the number of users. This has motivated the use of low complexity linear and decision driven suboptimal multiuser detection techniques [4]. In [10], Aazhang and Paris improve the performance of MUD in CDMA system using NN. Here NN is trained for the demodulation of signals via back-propagation type algorithm. Kechriotis and Manolakos have implemented an optimal CDMA multiuser detector using Hopfield NN in [11]. Also the performance of Hopfield $\mathrm{NN}$ receiver is better compared to any other suboptimal receiver. A fast training algorithm for ANN using feed forward multilayer perceptron architecture is proposed by Valadon and Tafazolli in [12]. The application of this algorithm to the problem of multiuser detection in the synchronous direct-sequence code-division multiple access (DS-CDMA) channel is investigated.

J. W. Hsun Kao et al [13] propose a blind multiuser detector using a machine learning technique called support vector machine (SVM) on a chaos-based CDMA system. Simulation results show that the performance achieved using SVM is comparable to existing MMSEC detector under both additive white gaussian noise (AWGN) and Rayleigh fading conditions. S. Chen et al [14] propose an additive training of NN using some stochastic gradient algorithm that aims to minimize the mean square error (MSE). This method developes a nonlinear adaptive near minimum error rate algorithm called the nonlinear least bit error rate (NLBER) for the training of NN. Multilayer perceptron based NN receiver architecture for the recovery of the information bits of a DS-CDMA users is proposed by Matyjas et al in [15]. Here a fast converging adaptive training algorithm is developed that minimizes BER. A recurrent $\mathrm{NN}$ for solving the nonlinear optimization problem involved in multiuser detection in CDMA is proposed by S. Liu and J. Wang in [16]. Compared with other existing works, the proposed NN globally converges to the exact optimal solution of the nonlinear optimization problem with nonlinear constraints and has relatively low structural complexity.

Review works reveal the fact that the joint capacity maximization and BER minimization in multiuser communication system is a non-convex integer problem and a closed form solution is difficult to find. Suboptimal solutions are proposed in many cases. Moreover, majority of the above algorithms suffer from exponential computation cost with the increase of number of subcarriers and users. Attempt is also made for some of these works to reduce the computation complexity from exponential to linear with the number of subcarriers (users) in OFDM (CDMA) system. However, when these concepts are used for implementation in MC-CDMA system, power allocations are not efficient due to the presence of in-band MAI. Hence, development of an efficient power allocation algorithm with low computation cost for multiuser multicarrier system is highly demanding.

It is also seen from the review works that the use of different soft-computing tools like GAs, ANN, fuzzy logic (FL) etc. offer low cost, tractable, ease of implementation and optimal solutions in designing optimized communication system. To this aim, GA-NN 
hybridization may be used to design optimized and adaptive system through learning/training. In brief, the contributions of the work are as follows: an efficient power allocation scheme in $\mathrm{CI} / \mathrm{MC}-\mathrm{CDMA}$ system is proposed using GA-NN hybridization under power constraint scenario. GA is used for optimal power allocation, while $\mathrm{NN}$ is used to calculate weight factors in MMSEC receiver. Stable decision variables so obtained enable better user grouping that leads to efficient block PIC. The objective function of GA is developed from the weighted average of channel capacity and minimum transmission power. On the other hand, tan-sigmoid transfer function is used in NN. Performance of the proposed work highlights the relative merits and demerits with respecting to the existing related works.

\section{System Model}

MC-CDMA was first proposed in [17] and is a combination of CDMA and OFDM with the spreading codes applied in frequency domain. CI/MC-CDMA is an MC-CDMA scheme employing complex carrier interferometry $(\mathrm{CI})$ spreading codes. The CI code, for the $\mathrm{k}^{\text {th }}$ user,

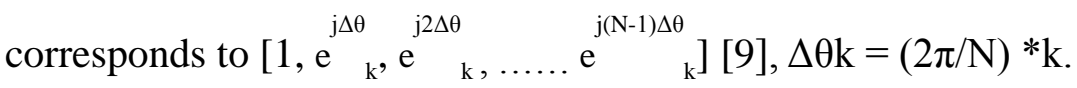

The CI codes [3] of length ' $\mathrm{N}$ ' have a unique feature that allows CI/MC-CDMA systems to support $\mathrm{N}$ users orthogonally. Then as system demand increases, codes can be selected to accommodate up to an additional ( $\mathrm{N}-1)$ users pseudo-orthogonally. Additionally, there is no restriction on the length ' $\mathrm{N}$ ' of the $\mathrm{CI}$ code (i.e., $\mathrm{N} \in \mathrm{I}$ ) making it more suitable for diverse wireless environments.

\subsection{Transmitter Model}

In CI/MC-CDMA transmitter [18], the incoming data $a_{k}$ for the $\mathrm{k}^{\text {th }}$ user, is transmitted over $\mathrm{N}$ narrowband subcarriers each multiplied with an element of the $\mathrm{k}^{\text {th }}$ user's spreading code. Binary phase shift keying (BPSK) modulation is assumed, i.e., $a_{k}= \pm 1$.

The mathematical form of total transmitted signal for $\mathrm{K}$ number of user is given in Eq. (1) below

$$
\mathrm{S}(\mathrm{t})=\sum_{k=1}^{K} \sum_{i=0}^{N-1} \mathrm{a}_{\mathrm{k}}[\mathrm{n}] \beta_{\mathrm{k}, \mathrm{i}} \operatorname{Cos}\left(2 \pi \mathrm{f}_{\mathrm{i}} \mathrm{t}+\mathrm{i} \Delta \theta_{\mathrm{k}}\right) * \mathrm{P}(\mathrm{t})
$$

where $f_{i}=f_{c}+i \Delta f$ and $P(t)$ is a rectangular pulse of duration $T_{b}$. The parameter $\beta_{k, i}$ is the amplitude of $k^{\text {th }}$ user at $i^{\text {th }}$ subcarrier which controls the transmitted power. Subcarrier spacing $\Delta \mathrm{f}$ is selected such that the carrier frequencies $\mathrm{f}_{\mathrm{i}}, \mathrm{i}=0,1, \ldots \mathrm{N}-1$ are orthogonal to each other, i.e. $\Delta \mathrm{f}=1 / \mathrm{T}_{\mathrm{b}}$.

\subsection{Channel Model}

An uplink model has been considered where all the user's transmissions are synchronized. It is assumed that every user experiences an independent propagation environment that is modeled as a slowly varying multipath channel. Multipath propagation in 
time translates into frequency selectivity in the frequency domain [4]. Frequency selectivity refers to the selectivity over the entire bandwidth of transmission and not over each subcarrier transmission. This is because $1 / \mathrm{T}_{\mathrm{b}}<<\left(\Delta \mathrm{f}_{\mathrm{C}}\right)<\mathrm{BW}$ where, $\Delta \mathrm{f}_{\mathrm{C}}$ is the coherence bandwidth and $\mathrm{BW}$ is the total bandwidth.

\subsection{Receiver Model}

The received signal without dispreading operation is written in Eq.(2) given below

$$
\mathrm{r}(\mathrm{t})=\sum_{k=1}^{K} \sum_{i=0}^{N-1} \mathrm{a}_{\mathrm{k}}[\mathrm{n}] \beta_{\mathrm{k}, \mathrm{i}} \alpha_{\mathrm{k}, \mathrm{i}} \operatorname{Cos}\left(2 \pi \mathrm{f}_{\mathrm{i}} \mathrm{t}+\mathrm{i} \Delta \theta_{\mathrm{k}}+\Phi_{\mathrm{k}, \mathrm{i}}\right) * \mathrm{P}(\mathrm{t})+\eta(\mathrm{t})
$$

where $\alpha_{\mathrm{k}, \mathrm{i}}$ is the Rayleigh fading gain and $\Phi_{\mathrm{k}, \mathrm{i}}$ is uniformly distributed phase offset of the $\mathrm{k}^{\text {th }}$ user in the $\mathrm{i}^{\text {th }}$ carrier and $\eta(\mathrm{t})$ represents additive white gaussian noise (AWGN). The received signal is projected onto $\mathrm{N}$ orthogonal carriers and is dispread using $\mathrm{j}^{\text {th }}$ users $\mathrm{CI}$ code resulting in $\mathrm{r}^{\mathrm{j}}=\left(\mathrm{r}_{0}{ }_{0}, \mathrm{r}_{1}{ }_{1}, \mathrm{r}_{2}{ }_{2}, . \mathrm{r}_{\mathrm{N}-1}^{\mathrm{j}}\right)$. The term $\mathrm{r}_{\mathrm{i}}^{\mathrm{j}}$ is shown in Eq. (3) below

$$
\mathrm{r}_{\mathrm{i}}^{\mathrm{j}}=\mathrm{a}_{\mathrm{j}}[\mathrm{n}] \beta_{\mathrm{j}, \mathrm{i}} \alpha_{\mathrm{j}, \mathrm{i}}+\sum_{k=1, k \neq j}^{K} \mathrm{a}_{\mathrm{k}}[\mathrm{n}] \beta_{\mathrm{k}, \mathrm{i}} \alpha_{\mathrm{k}, \mathrm{i}} \operatorname{Cos}\left(\mathrm{i}\left(\Delta \theta_{\mathrm{k}}-\Delta \theta_{\mathrm{j}}\right)+\Phi_{\mathrm{k}, \mathrm{i}}-\Phi_{\mathrm{j}, \mathrm{i}}\right)+\eta_{\mathrm{j}}
$$

where $n_{j}$ is a gaussian random variable with mean 0 and variance $\mathrm{N}_{0} / 2$. Now, a suitable combining strategy is used to create a decision variable $D_{j}$, which then enters a decision device that outputs $\hat{a}_{j}$. MMSEC is employed as it is shown to provide the best performance in a frequency selective fading channel [19].

The decision variable $\mathrm{D}_{\mathrm{j}}$ corresponds to [18]

$$
\mathrm{D}_{\mathrm{n}}^{\mathrm{j}}=\sum_{i=1}^{N} \mathrm{r}_{\mathrm{i}}^{\mathrm{j}} \mathrm{W}_{\mathrm{j}, \mathrm{i}}
$$

where

$$
\mathrm{W}_{\mathrm{j}, \mathrm{i}}=\frac{\alpha_{j, i} * \beta_{j, i}}{\left(\operatorname{Var}\left(a_{j}\right) A_{j, i}+\frac{N_{0}}{2}\right)}
$$

and

$$
\begin{aligned}
& \mathrm{A}_{\mathrm{ji}}=\sum_{k=1, k \neq j}^{K} \beta_{\mathrm{k}, \mathrm{i}}^{2} \alpha_{\mathrm{k}, \mathrm{i}}^{2} \operatorname{Cos}\left(\mathrm{i}\left(\left(\Delta \theta_{\mathrm{k}}-\Delta \theta_{\mathrm{j}}\right)+\Phi_{\mathrm{k}, \mathrm{i}}-\Phi_{\mathrm{j}, \mathrm{i}}\right)^{2}\right. \\
& \text { with } \operatorname{Var}\left(\mathrm{a}_{\mathrm{j}}\right)=1 .
\end{aligned}
$$

Thus, the outputs of all the single user detection of all users generate a decision vector $\mathrm{D}=\left[\mathrm{D}^{1}, \mathrm{D}^{2}, \ldots \ldots \mathrm{D}^{\mathrm{K}}\right]$ which is used to obtain the initial estimates of the data $a^{\wedge}=\left[\hat{a}_{1}, \hat{a}_{2}, \ldots . . \hat{a}_{k}\right]$. These initial estimates are then used to evaluate the MAI experienced by each user in the interference cancellation techniques [20]. Eq. (4) and (5) are effective when CSI is available. However in many cases CSI estimation in real time operation is 
computationally expensive and may be outdated due to feedback delay. One possible solution may be to calculate weight factor in eq. (4) through learning and may be used for blind detection.

\subsection{Antenna Diversity}

Diversity using two antennas, for simplicity, is used here although it can be extended for multiple antenna systems to achieve improved performance at the cost of greater computation. Received signal from two antennas are then weighted averaged according to their individual SIR. This diversity technique has the advantage of producing an acceptable weighted SIR even when none of the individual SIRs are themselves acceptable.

The received signal from antenna system 1, derived from equation (2) is rewritten here as follows for further analysis. Subscript ' 1 ' stands for antenna system 1 .

$$
\mathrm{r}(\mathrm{t})_{1}=\sum_{k=1}^{K} \sum_{i=0}^{N-1} \mathrm{a}_{\mathrm{k}}[\mathrm{n}] \beta_{\mathrm{k}, \mathrm{i}} \alpha_{\mathrm{k}, \mathrm{i}} \operatorname{Cos}\left(2 \pi \mathrm{f}_{\mathrm{i}} \mathrm{t}+\mathrm{i} \Delta \theta_{\mathrm{k}}+\phi_{\mathrm{k}, \mathrm{i}}\right) * \mathrm{P}(\mathrm{t})+\eta(\mathrm{t})
$$

So the received signal for the $\mathrm{j}^{\text {th }}$ user may be written as

$$
\begin{gathered}
\mathrm{r}_{\mathrm{j}}=\mathrm{a}_{\mathrm{j}}[\mathrm{n}] \beta_{\mathrm{j}, \mathrm{i}} \alpha_{\mathrm{j}, \mathrm{i}}+\sum_{k=1, k \neq j}^{K} \sum_{i=0}^{N-1} \mathrm{a}_{\mathrm{k}}[\mathrm{n}] \beta_{\mathrm{k}, \mathrm{i}} \alpha_{\mathrm{k}, \mathrm{i}} \operatorname{Cos}\left(\mathrm{i}\left(\Delta \theta_{\mathrm{k}}-\Delta \theta_{\mathrm{j}}\right)+\phi_{\mathrm{k}, \mathrm{i}}-\phi_{\mathrm{j}, \mathrm{i}}\right)+\eta_{\mathrm{j}, \mathrm{i}} \\
=\mathrm{a}_{\mathrm{j}}[\mathrm{n}] \beta_{\mathrm{j}, \mathrm{i}} \alpha_{\mathrm{j}, \mathrm{i}}+\mathrm{I}_{\mathrm{MAI}}+N
\end{gathered}
$$

The first term of Eq. (6b) is desired signal term, while the second and the third terms are MAI and AWGN term, respectively. Multiple access interference IMAI

$$
\begin{gathered}
\mathrm{I}_{\mathrm{MAI}}=\sum_{k=1, k \neq j}^{K} \sum_{i=0}^{N-1} \mathrm{a}_{\mathrm{k}}[\mathrm{n}] \beta_{\mathrm{k}, \mathrm{i}} \alpha_{\mathrm{k}, \mathrm{i}} \operatorname{Cos}\left(\mathrm{i}\left(\Delta \theta_{\mathrm{k}}-\Delta \theta_{\mathrm{j}}\right)+\phi_{\mathrm{k}, \mathrm{i}}-\phi_{\mathrm{j}, \mathrm{i}}\right) \\
=\left[\mathrm{I}_{\text {MAI }}^{1}, \mathrm{I}_{\text {MAI }}^{2} \ldots \ldots \ldots \ldots \ldots \ldots \mathrm{I}_{\mathrm{MAI}}^{\mathrm{k}}\right] \text { and the noise term } N=\left[\mathrm{n}_{1 \mathrm{j}}, \mathrm{n}_{2 \mathrm{j}} \ldots \ldots \ldots \ldots \ldots \mathrm{n}_{\mathrm{kj}}\right](8)
\end{gathered}
$$

For the Rayleigh fading channel and the large value of ' $K$ ' i.e. the number of users, the distribution of MAI is approximately Gaussian (according to central limit theorem). Noise $\mathbf{N}$ is also a Gaussian random variable with mean 0 and variance $\mathrm{N}_{0} / 2$. Therefore the interference and noise power is equal to variance of the interference and noise terms of all the users. Now the interference power and noise power, shown in Eq. (9) and (10), respectively can be expressed as follows:

and

$$
\sigma^{2}{ }_{\text {MAI }}=\operatorname{Var}\left(\mathrm{I}_{\mathrm{MAI}}\right)
$$

$$
\sigma^{2}{ }_{N}=\operatorname{Var}(N)
$$

The SIR for the $\mathrm{j}^{\text {th }}$ user of antenna system 1is shown in Eq.(11) below

$$
\operatorname{SIR}_{\mathrm{j} 1}=\mathrm{a}^{2}{ }_{\mathrm{j}}[\mathrm{n}] * \alpha_{\mathrm{j}, \mathrm{i}}^{2} * \beta_{\mathrm{j}, \mathrm{i}} /\left(\sigma^{2}{ }_{\mathrm{MAI}}+\sigma^{2}{ }_{\mathrm{N}}\right)
$$


So the total SIR for the antenna system 1 can be written

$$
\mathrm{SIR}_{1}=\sum_{j=1}^{K} \mathrm{SIR}_{\mathrm{j} 1}
$$

Similarly total SIR for the antenna system 2 can be written

$$
\mathrm{SIR}_{2}=\sum_{j=1}^{K} \operatorname{SIR}_{\mathrm{j} 2}
$$

Total weighted SIR of two-antenna receiver system is shown in Eq. (12) below

$$
\mathrm{SIR}_{\mathrm{TW}}=\mathrm{W}_{1} * \mathrm{SIR}_{1}+\mathrm{W}_{2} * \mathrm{SIR}_{2}
$$

where $\mathrm{W}_{1}=10 * \mathrm{SIR}_{1} /\left(\mathrm{SIR}_{1}+\mathrm{SIR}_{2}\right)$, and $\mathrm{W}_{2}=10 * \mathrm{SIR}_{2} /\left(\mathrm{SIR}_{1}+\mathrm{SIR}_{2}\right)$

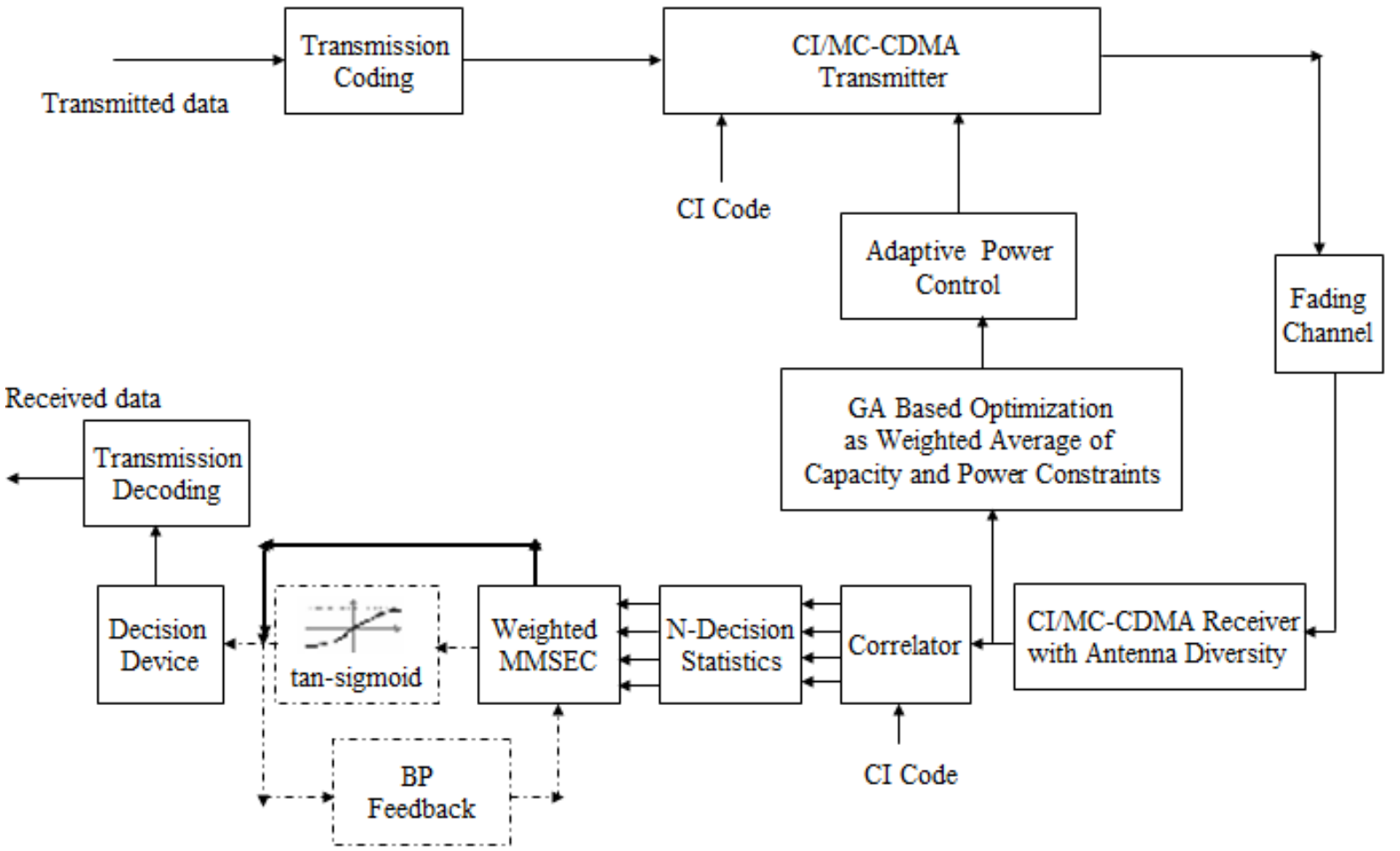

Fig.1 Block diagram for GA based power control and NN based weight calculation.

\section{Proposed GA Based Power Allocation Algorithm}

This section briefly describes proposed GA based adaptive power allocation for the antenna diversity assisted CI/MC-CDMA system described in previous section. The proposed system is also shown in Fig. 1 as block diagram representation. The main objective of this work is to maximize the overall channel capacity as well as maintaining BER performance 
within a limit under the constraint of transmit power. We first define objective function followed by GA implementation for power allocation.

\subsection{Formulation of Objective Function}

Wireless channel is highly nonlinear and random in nature. In the present system, it is assumed that during training process CSI is available in transmitter (feedback from receiver) and is used to modify signal amplitude so that the channel capacity is maximized and the probability of bit error is maintained within the limit, subject to transmit power constraints. Mathematically it is a nonlinear integer problem of conflicting nature and is difficult to find the close from solution. Hence, one sub-optimal solution may be used to find adaptive power for each user on each subcarrier. The fitness (objective) function is shown in Eq. (13) as follows

$$
\mathrm{F}=\lambda_{1} * \sum_{k=1}^{K} \sum_{i=0}^{N-1} \log _{2}\left[1+\frac{\alpha_{k, i}^{2} * p_{k, i}}{\left(\sigma^{2}{ }_{M A 1}+\sigma_{N}^{2}\right)}\right]+\lambda_{2} *\left(\sum_{k=1}^{K} \sum_{i=0}^{N-1} p_{k, i}-\mathrm{P}_{\text {total }}\right)
$$

subject to $b_{e} \leq B$ where $b_{e}$ is the probability of bit error i.e BER (bit error rate), B is threshold or upper limit an acceptable BER value, $\mathrm{p}_{\mathrm{k}, \mathrm{i}}, \mathrm{P}_{\text {total }}$ denote the transmitted power of $\mathrm{k}^{\text {th }}$ user on $\mathrm{i}^{\text {th }}$ subcarrier, and the maximum allowable transmit power, respectively. The symbols $\lambda_{1}$ and $\lambda_{2}$ are the weight factors such that $\lambda_{1}+\lambda_{2}=1$. We assume equal value for both the weight factor i.e. $\lambda_{1}=\lambda_{2}=0.5$. The expression of $\sigma_{\mathrm{MAI}}$ may be stated as follows: $\sigma_{\mathrm{MAI}}=\mathrm{f}$ $\left(\rho_{\mathrm{j}, \mathrm{k}}, \alpha_{\mathrm{k}, \mathrm{i}}, \beta_{\mathrm{k}, \mathrm{i}}\right)$ where $\rho_{\mathrm{j}, \mathrm{k}}$ is the cross-correlation between $\mathrm{j}^{\text {th }}$ and $\mathrm{k}^{\mathrm{th}}$ users. Incorporating the correlation coefficient $\rho$, we may write the interference power $\sigma_{\text {MAI }}^{2}=\rho^{2} * \alpha_{k, i}^{2} * \beta_{k, i}^{2}$ and noise power $\sigma^{2}{ }_{\mathrm{N}}=\mathrm{N}_{0}$. Putting these values in eq. (13), we get fitness function

$$
\left.\mathrm{F}=\lambda_{1} * \sum_{k=1}^{K} \sum_{i=0}^{N-1} \log _{2}\left[1+\frac{\alpha_{k, i}^{2} * \beta_{k, i}^{2}}{\left(\rho^{2} * \alpha_{k, i}^{2} * \beta_{k, i}^{2}+N_{0}\right.}\right)\right]+\lambda_{2} *\left(\sum_{k=1}^{K} \sum_{i=0}^{N-1} \beta_{k, i}^{2}-\mathrm{P}_{\text {total }}\right)
$$

Our goal is to maximize eq. (14) subject to $b_{e} \leq B$.

\subsection{GA Based Adaptive Power Allocation}

Genetic algorithm (GA) is one of the robust global optimization tools widely explored in solving complex optimization problems in numerous fields. The operations of GA depend on initial population, crossover and mutation. In the present study, adaptive power of each user on each subcarrier is calculated based on maximization of fitness function $\mathrm{F}$ defined in eq. (14). The experimental conditions of GA for the present problem are depicted as follows: size of population is 20 , number of generations 100 , probability of crossover per generation is 0.8 , and probability of mutation per bit is 0.09 , upper limit set on MMSEC receiver's BER value i.e. $B=10^{-2}$. Different steps for implementing GA based adaptive transmitter power allocation are described as follows: 
Step 1: Initialization of twenty sets of random values of transmitted power within the maximum limit allowed by the power constraint channel.

Step 2: Calculate the fitness value $F$ for the twenty sets of random transmit power obtained in step 1. A predefined threshold $\left(F_{u}\right)$ value of $F$ is assigned to identify the fit parameter sets.

Step 3: The particular set of transmit powers which produce the fitness value $F$ above $\left(F_{u}\right)$ are duplicated and the remaining sets are ignored from the population.

Step 4: A set of binary string is generated through decimal-to-binary conversion of all selected transmitted powers. Now crossover and mutation operation are done according to their respective probabilities stated above.

Step 5: A new set of transmitted power within the range are generated through binary-to-decimal conversion of the strings obtained in step 4.

Step 6: Repeat step 1 to step 5 for the desired number of iterations or till a predefined acceptable values for channel capacity and BER $\left(b_{e}\right)$ are achieved. Fig. 2 shows the flow diagram of the proposed GA based adaptive power allocation. 


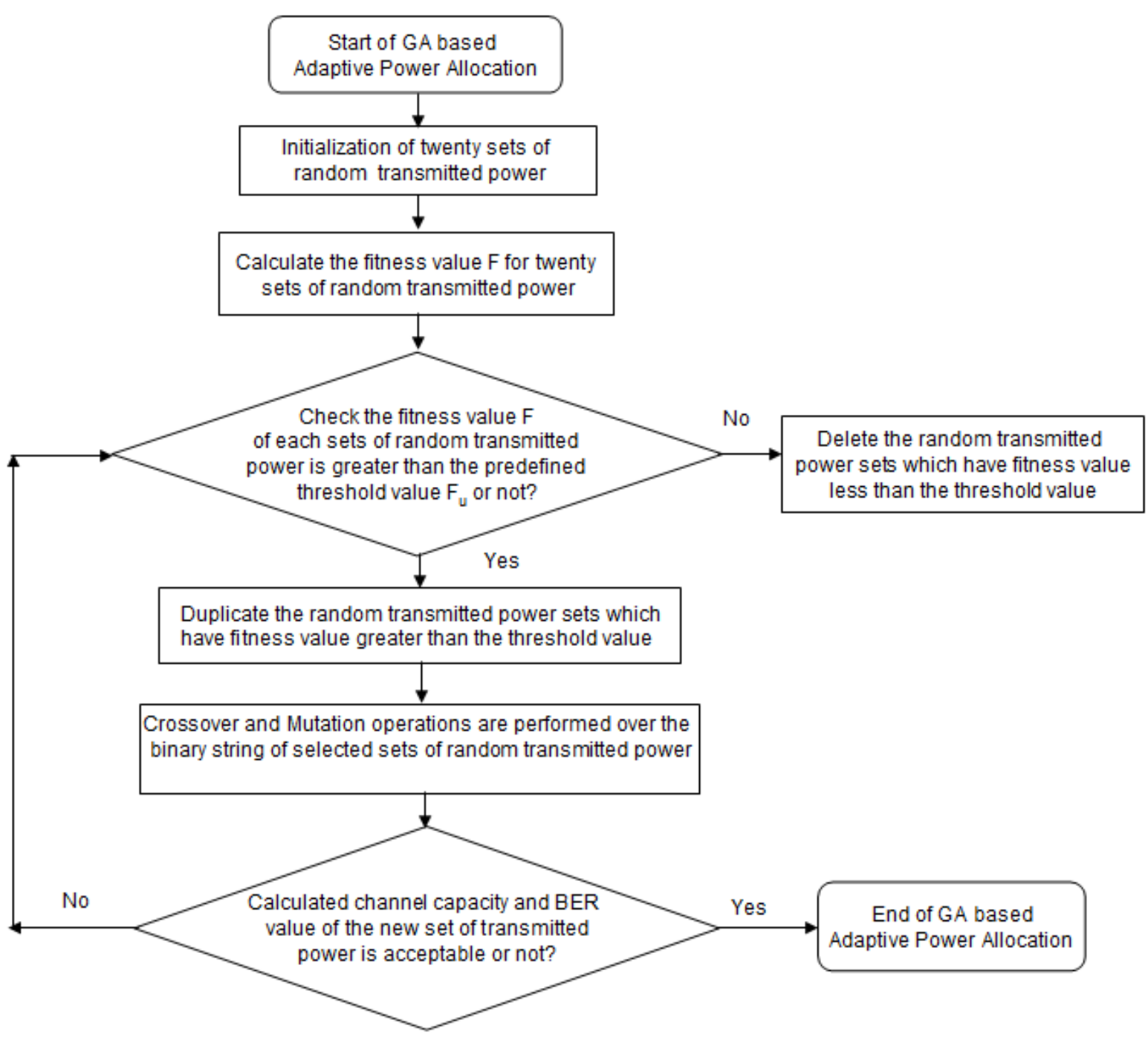

Fig.2 Flow diagram for GA based Adaptive Power Allocation.

\section{Neural Network Assisted PIC Model}

Parallel interference cancellation (PIC) is a method to decrease the multiple access interference (MAI) by cancelling the effect of the other users. In this paper, we compare the BER values between conventional PIC and PIC with neural network. The received signal is projected onto $\mathrm{N}$ orthogonal carriers and is dispread using $\mathrm{j}^{\text {th }}$ users $\mathrm{CI}$ code resulting in $r_{j}=\left(r_{j}^{0}, r_{j}^{1}, r_{j}^{2}, \ldots . . r_{j}^{N-1}\right)$, where $r_{j}^{i}$ corresponds to Eq. (3) is rewritten here for convenience of analysis

$$
\mathrm{r}_{\mathrm{i}}^{\mathrm{j}}=\mathrm{a}_{\mathrm{j}}[\mathrm{n}] \beta_{\mathrm{j}, \mathrm{i}} \alpha_{\mathrm{j}, \mathrm{i}}+\sum_{k=1, k \neq j}^{K} \mathrm{a}_{\mathrm{k}}[\mathrm{n}] \beta_{\mathrm{k}, \mathrm{i}} \alpha_{\mathrm{k}, \mathrm{i}} \operatorname{Cos}\left(\mathrm{i}\left(\Delta \theta_{\mathrm{k}}-\Delta \theta_{\mathrm{j}}\right)+\Phi_{\mathrm{k}, \mathrm{i}}-\Phi_{\mathrm{j}, \mathrm{i}}\right)+\eta_{\mathrm{j}}
$$

The first term of eq. (15) is the data of $\mathrm{j}^{\text {th }}$ user at $\mathrm{i}^{\text {th }}$ subcarrier, the second term is MAI 
due to the users other than $\mathrm{j}^{\text {th }}$ user and the third term is noise term for additive white Gaussian noise spread by $\mathrm{j}^{\text {th }}$ user code pattern.

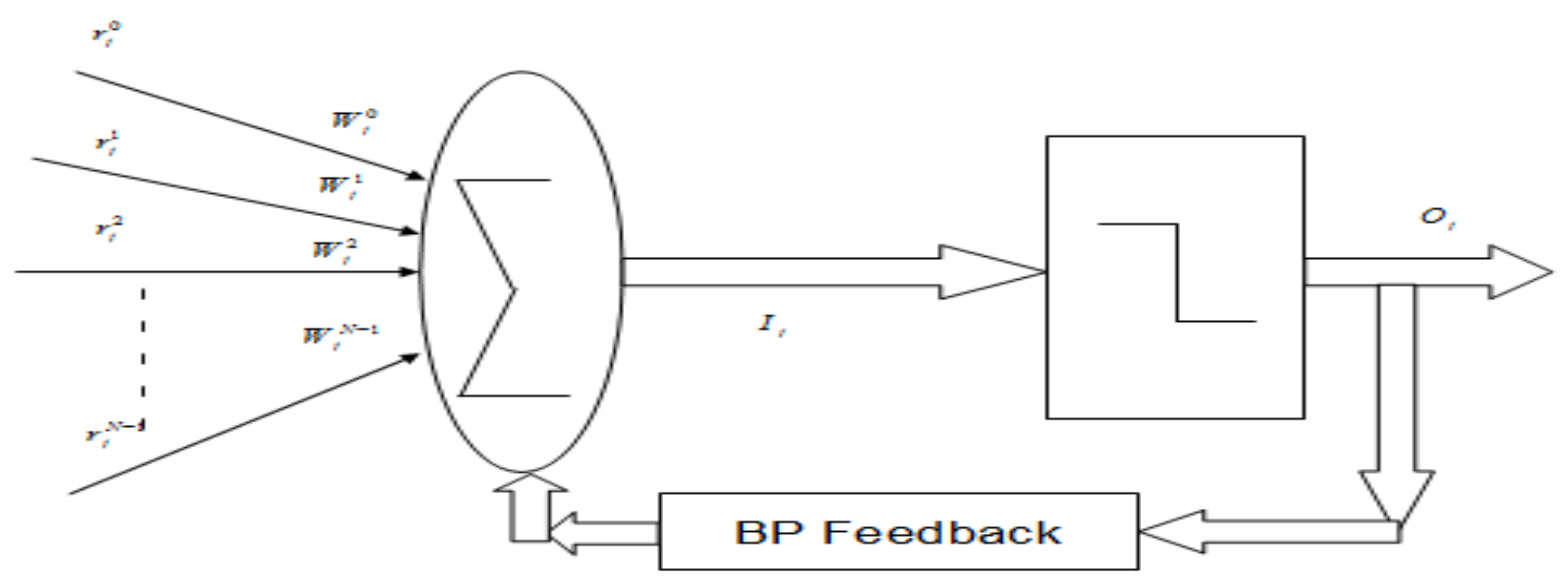

Fig. 3 Neural network based amplitude estimation for $\mathrm{j}^{\text {th }}$ user.

Here $r_{j}^{i}$ is the input to $\mathrm{NN}$ and $W_{j}^{i}$ is the weight factor of $\mathrm{j}^{\text {th }}$ user at $\mathrm{i}^{\text {th }}$ subcarrier as shown in Fig. 3. So the output of summation unit $I_{j}$ (similar to eq. (4)) is shown in Eq. (16) below

$$
\begin{aligned}
& I_{j}=\sum_{i=0}^{N-1} W_{j}^{i} * r_{j}^{i}=\sum_{i=0}^{N-1} W_{j}^{i} *\left[a_{j} \beta_{j, i} \alpha_{j, i}+\sum_{k=1, k \neq j}^{K} a_{k} \beta_{k, i} \alpha_{k, i} \operatorname{Cos}\left(i\left(\Delta \theta_{k}-\Delta \theta_{j}\right)+\phi_{k, i}-\phi_{j, i}\right)+\eta_{j}(s)\right]= \\
& =\sum_{i=0}^{N-1} W_{j}^{i} * a_{j} \beta_{j, i} \alpha_{j, i}+\sum_{i=0}^{N-1} I_{O S}+\sum_{i=0}^{N-1} I_{N S}
\end{aligned}
$$

where $I_{O S}=W_{j}^{i} * \sum_{k=1, k \neq j}^{K} a_{k} \beta_{k, i} \alpha_{k, i} \operatorname{Cos}\left(i\left(\Delta \theta_{k}-\Delta \theta_{j}\right)+\phi_{k, i}-\phi_{j, i}\right)$ is the MAI due to the users other than $\mathrm{j}^{\text {th }}$ user and $I_{N S}=W_{j}^{i} * \eta_{j}$ is the interference due to noise. Note that Eq. (16) contains three terms: desired signal term, MAI and noise term, respectively as in Eq. (7b).

The output of NN for the $\mathrm{j}^{\text {th }}$ user is $O_{j}$. Now we have to apply back propagation algorithm (BP) to adjust the weight factors $W_{j}^{0}, W_{j}^{1}, \ldots \ldots W_{j}^{N-1}$ on different subcarriers.

The target output information for the $\mathrm{j}^{\text {th }}$ user, $T_{j}=\beta_{j} \alpha_{j}$. But the computed output for the $\mathrm{j}^{\text {th }}$ user by the neuron is $O_{j}=\hat{\beta}_{j} \hat{\alpha}_{j}$

Mathematical expression of error signal is given in Eq. (17) below 


$$
E_{j, i}=\frac{1}{2}\left[T_{j}-O_{j}\right]^{2}=\frac{1}{2}\left[\beta_{j} \alpha_{j}-\hat{\beta}_{j} \hat{\alpha}_{j}\right]^{2}
$$

In a particular training session, the error $\mathrm{E}$ can be written as a function of the correlation values $r$ between the received signal and the signature/spreading waveform and weight factor W like the following

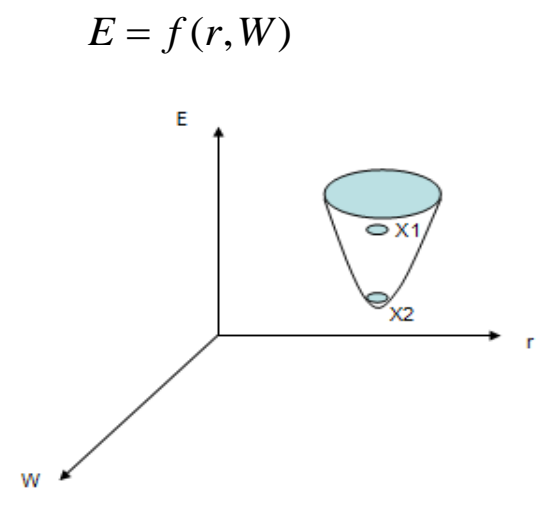

Fig. 4 A hypothetical error function.

Fig. 4 shows the plot of a hypothetical error function E. Let us assume that the point $\mathrm{X}_{1}$ denotes an error in prediction corresponding to a set of initial weights. The aim of the training session is to reach point $\mathrm{X}_{2}$, at which the error in prediction $\mathrm{E}$ reaches its minimum value.

In back propagation algorithm, the error $\mathrm{E}$ is minimized using steepest gradient descend method, where the changes in weight factor values can be obtained as follows [21] $\Delta W=-\eta \frac{\partial E}{\partial W}$ where $\eta$ represents the learning rate lying between 0 to 1 . It is known as the

Delta Rule. It is important to mention that the smaller the value of $\eta$, the slower will be the rate of convergence resulting into a smoother network. On the other hand, a higher value of $\eta$ will make the convergence faster but the resulting network may become unstable.

To update the connecting weight for $\mathrm{i}^{\text {th }}$ subcarrier, the following relation may be written as

$$
\mathrm{W}_{\mathrm{i}, \text { Updated }}=\mathrm{W}_{\mathrm{i}, \text { Previous }}+\Delta \mathrm{W}_{\mathrm{i}}
$$

where the change in weight factor can be determined as

$$
\Delta W_{i}=-\eta \frac{\partial E_{j}}{\partial W_{i}}
$$

Eq. (18) and (19) indicate updated weight and change in weight.

Now, $\frac{\partial E_{j}}{\partial W_{i}}$ can be computed using the chain rule of differentiation as given in Eq. (20) 
below.

$$
\frac{\partial E_{j}}{\partial W_{i}}=\frac{\partial E_{j}}{\partial O_{j}} \frac{\partial O_{j}}{\partial I_{i}} \frac{\partial I_{j}}{\partial W_{i}}
$$

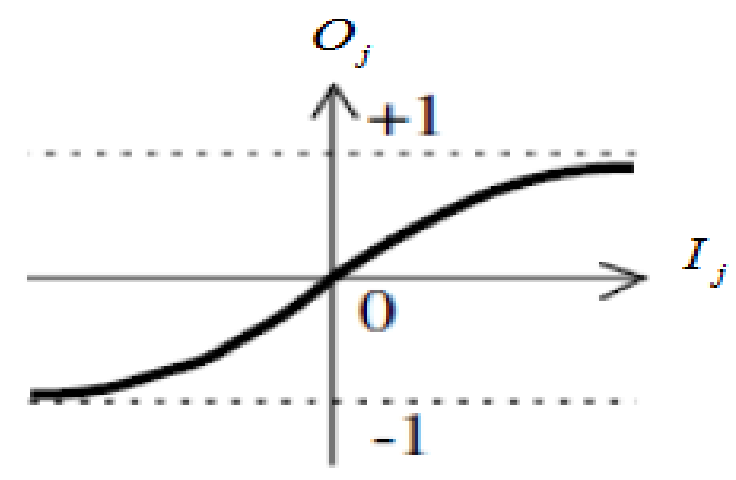

Fig. 5 Tan-sigmoid transfer function

Actually $O_{j}$ is the final output of the neuron obtained after passing through a non linear filter, known as activation function. As BPSK modulation is used, tan-sigmoid function is used here as transfer function as it produces values between -1 to 1 . Fig. 5 shows tan-sigmoid transfer function. So we have assumed that the neurons lying on the output layer to have tan-sigmoid transfer function.

The output of the $\mathrm{j}^{\text {th }}$ user neuron can be estimated as

$$
\left.O_{j}=\operatorname{tav} i g \lambda I_{j}\right)=\frac{e^{\lambda I_{j}}-e^{-\lambda I_{j}}}{e^{\lambda I_{j}}+e^{-\lambda I_{j}}}
$$

where $\lambda$ is the coefficient of the transfer function. Now from eq. (17), (21) and (16) we obtain

$$
\begin{aligned}
& \frac{\partial E_{j}}{\partial O_{j}}=-\left(T_{j}-O_{j}\right) \\
& \frac{\partial O_{j}}{\partial I_{i},}=\lambda\left(1+O_{j}\right)\left(1-O_{j}\right) \\
& \frac{\partial I_{j}}{\partial W_{i}}=r_{j}
\end{aligned}
$$

Substituting the values of $\frac{\partial E_{j,}}{\partial O_{j}}, \frac{\partial O_{i}}{\partial I_{i}}$ and $\frac{\partial I_{j}}{\partial W_{i}}$ in eq. (20), we get

$$
\frac{\partial E_{j}}{\partial W_{i}}=-\left(T_{j}-O_{j}\right) \lambda\left(1+O_{j}\right)\left(1-O_{j}\right) r_{j}
$$


Again, substituting the value of $\frac{\partial E_{j}}{\partial W_{i}}$ from Eq. (22d) in eq. (19), the change of weight factor $\Delta W_{i}$ can be determined as follows

$$
\Delta W_{i}=\eta \lambda\left(T_{j}-O_{j}\right)\left(1+O_{j}\right)\left(1-O_{j}\right) r_{j}
$$

Substituting the value of target output $T_{j}$ in eq. (24), we get the following equation

$$
\Delta W_{i}=\eta \lambda\left(\beta_{j} \alpha_{j}-O_{j}\right)\left(1+O_{j}\right)\left(1-O_{j}\right) r_{j}=\eta \lambda \beta_{j} \alpha_{j}\left(1+O_{j}\right)\left(1-O_{j}\right) r_{j}-\eta \lambda O_{i}\left(1+O_{j}\right)\left(1-O_{j}\right) r_{j}
$$

We obtain the rate of change of $\Delta W_{i}$ with respect to learning rate $(\eta)$ in Eq. (25) as follows

$$
\frac{\partial \Delta W_{i}}{\partial \eta}=\lambda \beta_{j} \alpha_{j}\left(1+O_{j}\right)\left(1-O_{j}\right) r_{j}\left(1-\frac{O_{j}}{T_{j}}\right)
$$

From the above equation, it may be stated that the rate of change of weight factor $\Delta W_{i}$ with learning rate $\eta$ is largely affected by term $\beta_{j} \alpha_{j}$. So stronger user's weight updation must be higher. Also when the output of neuron $O_{j}$ is equal to the target output $T_{j}$, then rate of change of $\Delta W_{i}$ with respect to learning rate $(\eta)$ will be zero this means no need to learn any thing more.

Also the updated value of weight factor can be easily obtained using $\Delta W_{i}$ from Eq. (23).

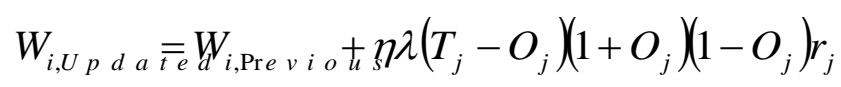

Based on this updated weight factor $\mathrm{W}_{\mathrm{j}, \mathrm{i}}$ in Eq. (26), we have calculated the BER performance using MMSEC strategy.

\subsection{Block PIC}

To achieve improved receiver performance through interference cancellation, block PIC (BPIC) is employed. This operation takes care the degrading effect of MAI. The decision variables $\left(D^{j}\right)$ are mapped to the interval $[0,1]$ through normalization i.e. dividing each $D^{j}$ by the maximum $\mathrm{D}$ value. The four BPIC method is described as follows.

1. The greater the magnitude of normalized decision variable $\left|D_{\text {normal }}^{j}\right|$, the stronger the 
interference effect of the respective users data. Thus, the users for which decision variables $\left|D_{n o r m a l}^{j}\right|$ corresponding to the magnitude of decisions variables satisfy the condition $0.75<\left|D_{\text {normal }}^{j}\right| \leq 1$ are classified as very strong user group. Similar rule is followed for the other users, the bits for which the decision variables satisfy the condition $0.50<\left|D_{\text {normal }}^{j}\right| \leq 0.75$, $0.25<\left|D_{\text {normal }}^{j}\right| \leq 0.50$ and $0<\left|D_{\text {normal }}^{j}\right| \leq 0.25$ are classified as strong, weak and very weak user groups, respectively.

2. BPIC is performed within the group of very strong user by simultaneously canceling the interference of all other user bits except the desired one and the very strong data bits are thus estimated.

3. Using the updated data of very strong users, the interferences due to these bits are removed and BPIC is employed within the block of strong user bits. The strong user's data are thus estimated.

4. Using the updated data of strong user's bits and very strong bits, the interferences due to these user's bits are removed. Then BPIC is employed within the group of weak users. The same processes are continued for all other remaining groups.

5. The updated decision statistics of the all user's bits are used to compute the $\left|D_{\text {normal }}^{j}\right|$ values. The BPIC process i.e. steps $1,2,3,4$ are repeated iteratively until a desired BER value for the decoded data is achieved or preset numbers of iterations are completed.

\section{Performance Evaluation}

This section presents performance evaluation of the proposed GA-NN hybridization for gain in channel capacity and BER performance. As the design goal was to show improved transmitter performance (channel capacity) using GA and receiver performance using NN, simulation results need to valid the claims. To this aim, performance evaluation for the adaptive synchronous CI/MC-CDMA system has been investigated via MATLAB simulation in terms of data transmission rate i.e. channel capacity vs number of users, comparison with [3] and [4], BER performance in MMSEC receiver and NN based BPIC system. Performance results are also reported for different combinations of the (non) adaptive systems, namely (i) non adaptive power and normal BPIC (without use of both GA and NN), (ii) adaptive power and normal BPIC (uses GA but without NN), (iii) non adaptive power with NN based BPIC (without GA but using NN), (iv) adaptive power control with NN based BPIC (using both GA and $\mathrm{NN}$ ). Simulations are done at $\mathrm{SNR}=7 \mathrm{~dB}$, number of users varying from 10 to 80 , the number of subcarrier is 24 and maximum allowable transmit power is 5 unit $(\mathrm{mW})$. 


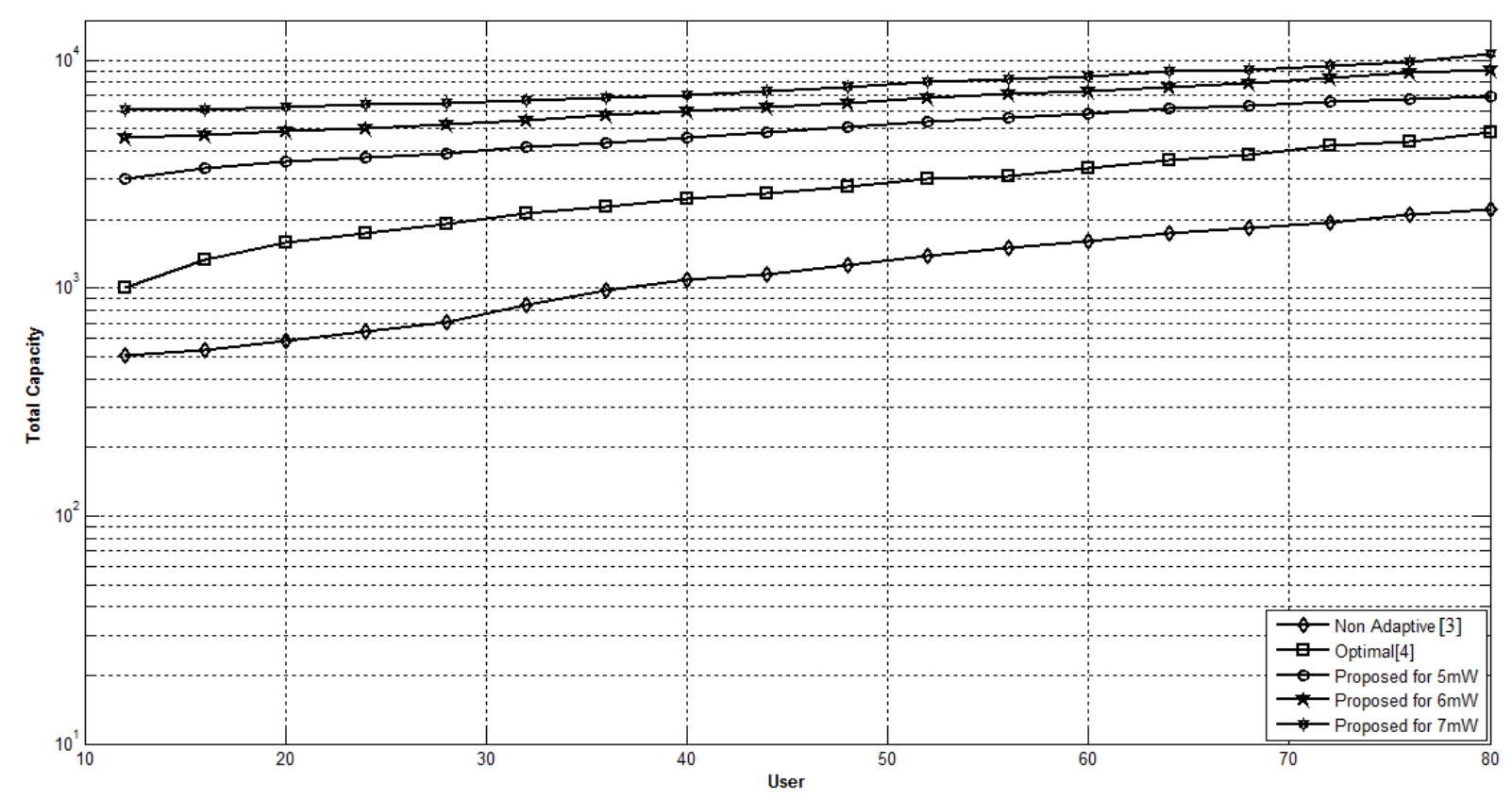

Fig.6 Performance comparison results for capacity vs. number of the users.

Fig.6 shows that the overall system channel capacity (data transmission rate) vs number of users. As expected, with the increase of number of users, sum capacity increases, however, this increase is higher in the proposed work than the existing methods [3] and [4]. It is also seen from the graphical results that channel capacity improvement in power adaptive system in [4] is significantly higher than the non power adaptive system [3]. This is due to the fact that power adaptive system takes into consideration the effect of MAI which in turn increase in SINR value. An improvement in channel capacity of the order of $\sim 300$ is achieved (vertical axis of the graph is plotted in semi log) in power adaptive system in [4] over non power adaptive system [3]. Furthermore, this channel capacity improvement is 200 order at user 80 for the proposed power adaptive system compared to [4] at $5 \mathrm{~mW}$ total transmission power. Channel capacity variation of the proposed system is also reported for total transmission power at $6 \mathrm{~mW}$ and $7 \mathrm{~mW}$. Simulation results also highlight that an increase $\sim 100$ order in capacity is achieved for an increase in $1 \mathrm{~mW}$ total transmission power. 


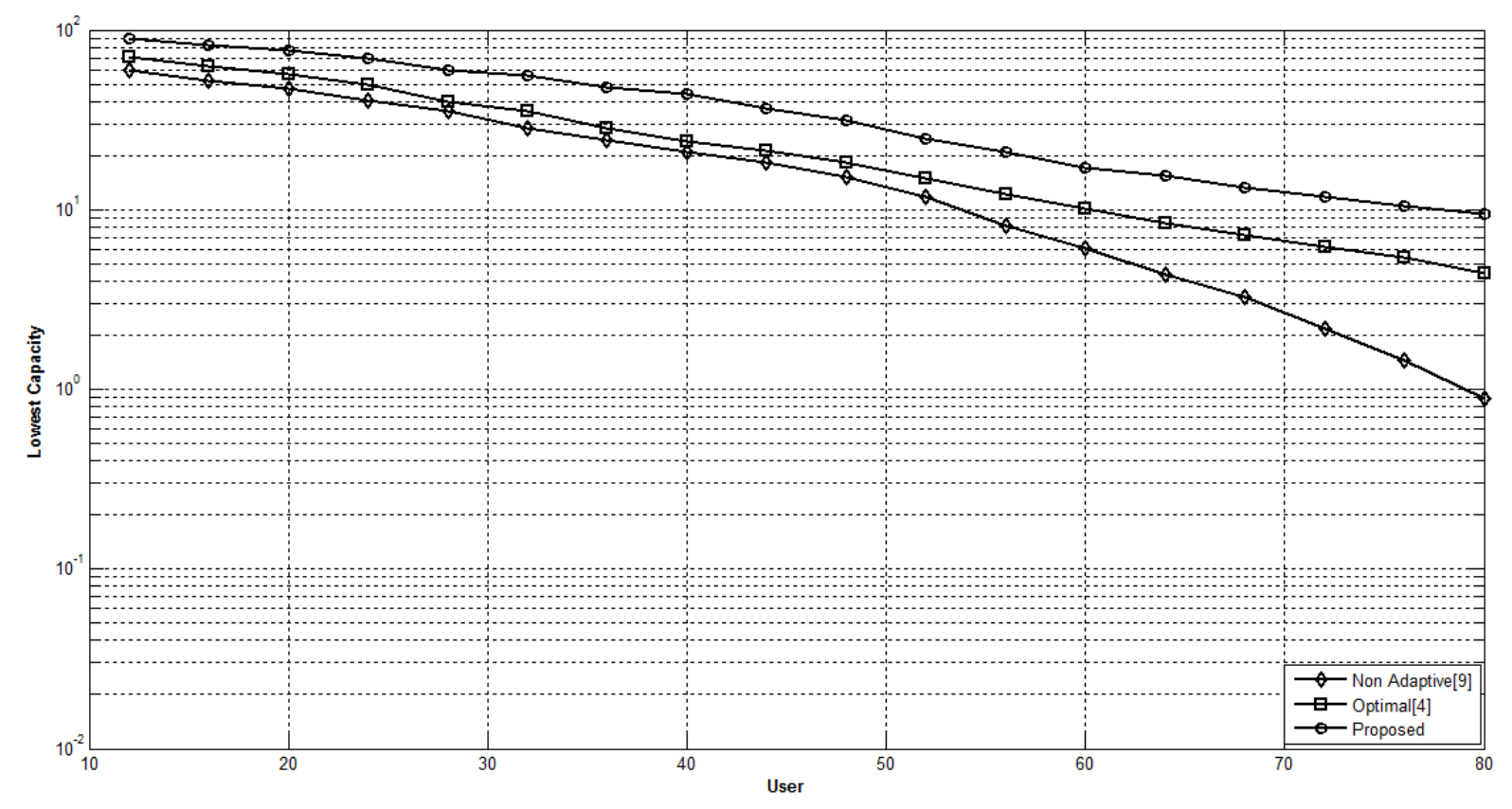

Fig.7 Performance comparison results for the lowest capacity vs. number of users.

Fig.7 shows that the lowest channel capacity of the worst user (data transmissions rate of the worst user i.e the user having worst fading gain) vs. number of users. As expected, with the increase of number of users, lowest channel capacity decreases due to MAI effect. Since the primary goal of this power allocation scheme is to increase SINR value, the overall lowest channel capacity is higher in the proposed work than the existing methods [3] and [4]. It is also seen from the graphical results that the lowest channel capacity improvement in power adaptive system in [4] is significantly higher than the non power adaptive system [3]. This is due to the fact that power adaptive system takes into consideration the effect of MAI which in turn increase in SINR value. An improvement in the lowest channel capacity of the order of 4 order is achieved (vertical axis of the graph is plotted in semi log) in power adaptive system in [4] over non power adaptive system [3]. Furthermore, this channel capacity improvement is $\sim 5$ order at user 80 for the proposed power adaptive system compared to [4] at $5 \mathrm{~mW}$ total transmission power. 


\section{Macrothink}

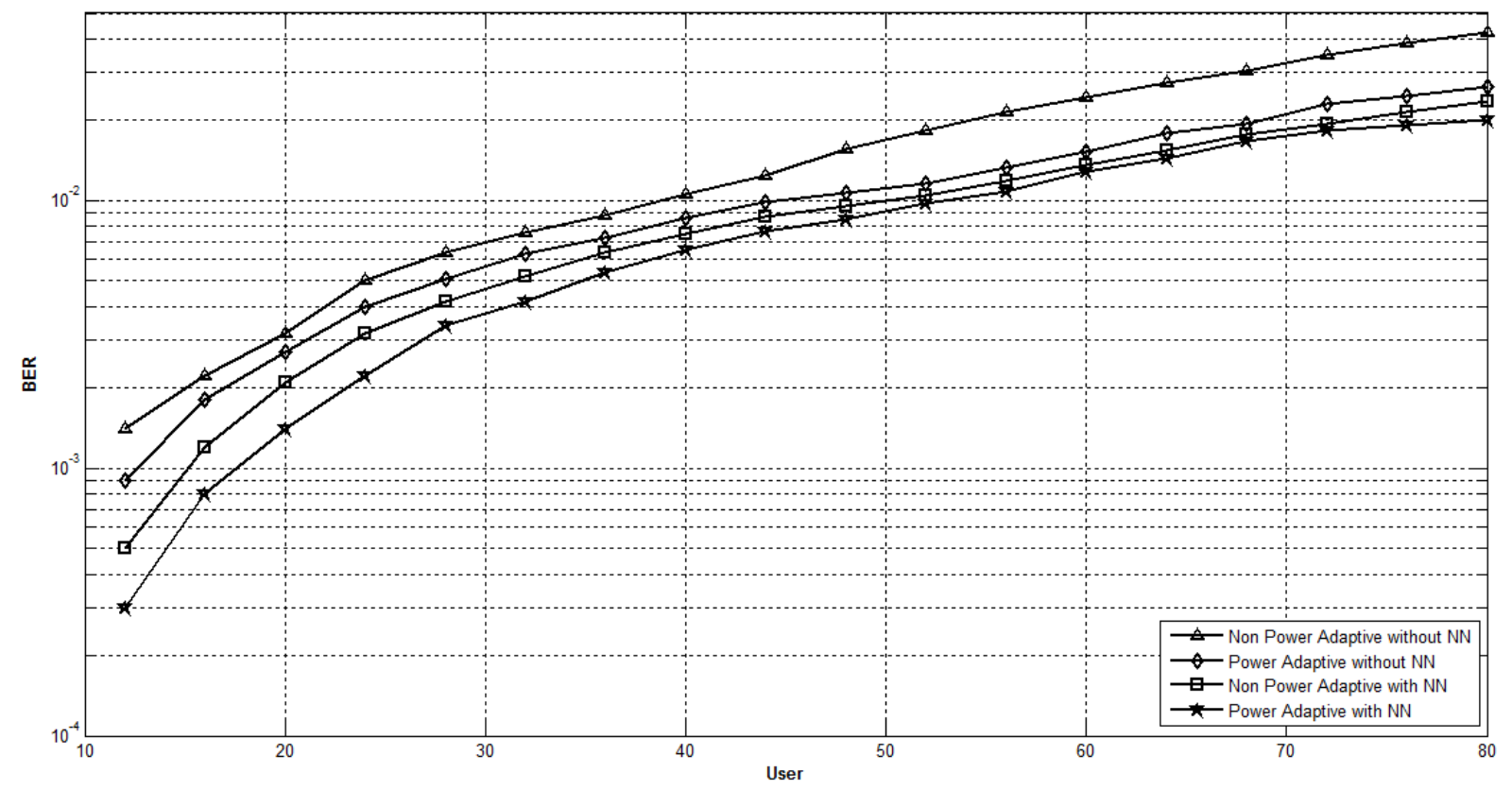

Fig.8 BER performance analysis for MMSEC receiver with number of users

Fig.8 represents BER performance for MMSEC receiver for power adaptive (using GA) and non adaptive system. MMSEC receiver is designed with and without using NN. Simulation results show that use of GA for power control and use of NN for weight calculation offer the best performance. On the other hand, without use of GA and NN, as expected, shows the worst BER performance. Since NN is used for efficient receiver design, non power adaptive but NN based MMSEC receiver offers relatively better BER performance compared to power adaptive (GA based) but non NN based MMSEC receiver. Simulation results also show that BER values for 80 users are 0.020 (for both GA-NN), 0.0235 (NN only), 0.0265 (GA only) and 0.0425 (without GA and NN). Simulation results highlight the importance of not only the adaptive power allocation but also improved/weighted correlator receiver. BER performance shows are obtained without using any error-control code (ECC), needless to maintain that BER values would be lowered significantly using ECC

It is seen that upper limit for BER value during simulation is set to $10^{-2}$ which is not always acceptable in many multimedia communication. To improve further BER performance several forms of multiuser detection in CDMA is reported like [4]. We finally integrate block PIC scheme in [20] with the proposed adaptive power allocation scheme to improve BER performance at high user capacity. In brief, users are classified into different groups, namely, very strong, strong, weak and very weak based on the magnitudes of decision statistics. The interferences within the individual groups of large decision magnitudes like very strong, strong, weak and very weak in order of sequence are removed to improve BER performance for the weakest group. 


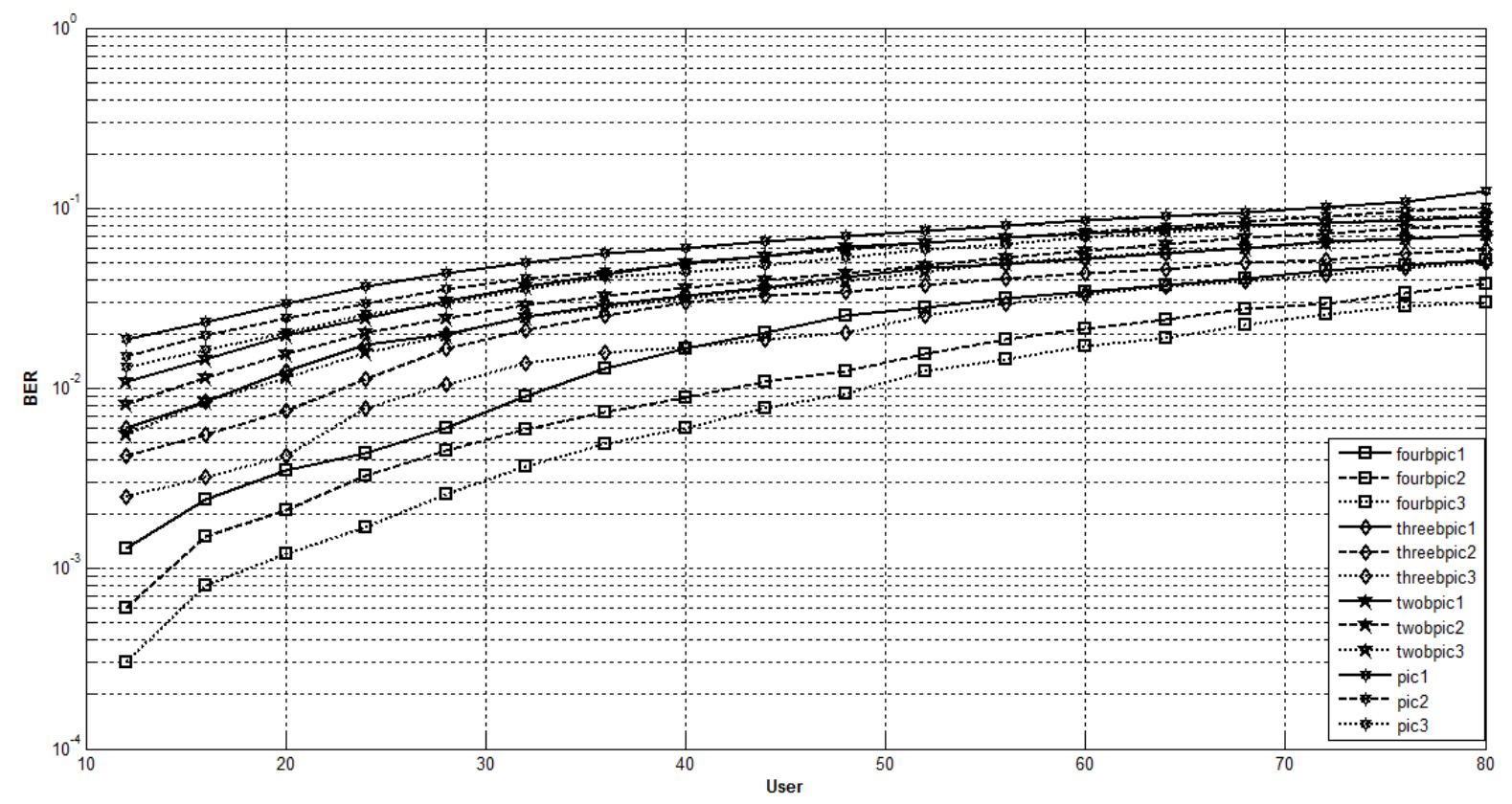

Fig.9 BER performance analysis for non-power adaptive normal BPIC

Fig.9 shows BER performance for the non power adaptive system (without use of GA and NN) but using BPIC. At greater number of users, BER at the initial stage being high, interference cancelation will not improve much in BER performance. Although BPIC scheme is employed but BER performance is not satisfactory due to MAI effect at subcarrier level. Multistage interference cancellation is used to reduce this MAI effect. An improvement in BER performance for users 80 is achieved from 0.1235 to 0.0300 at third stage interference cancellation of 4BPIC (4 group BPIC) over singe stage PIC scheme.

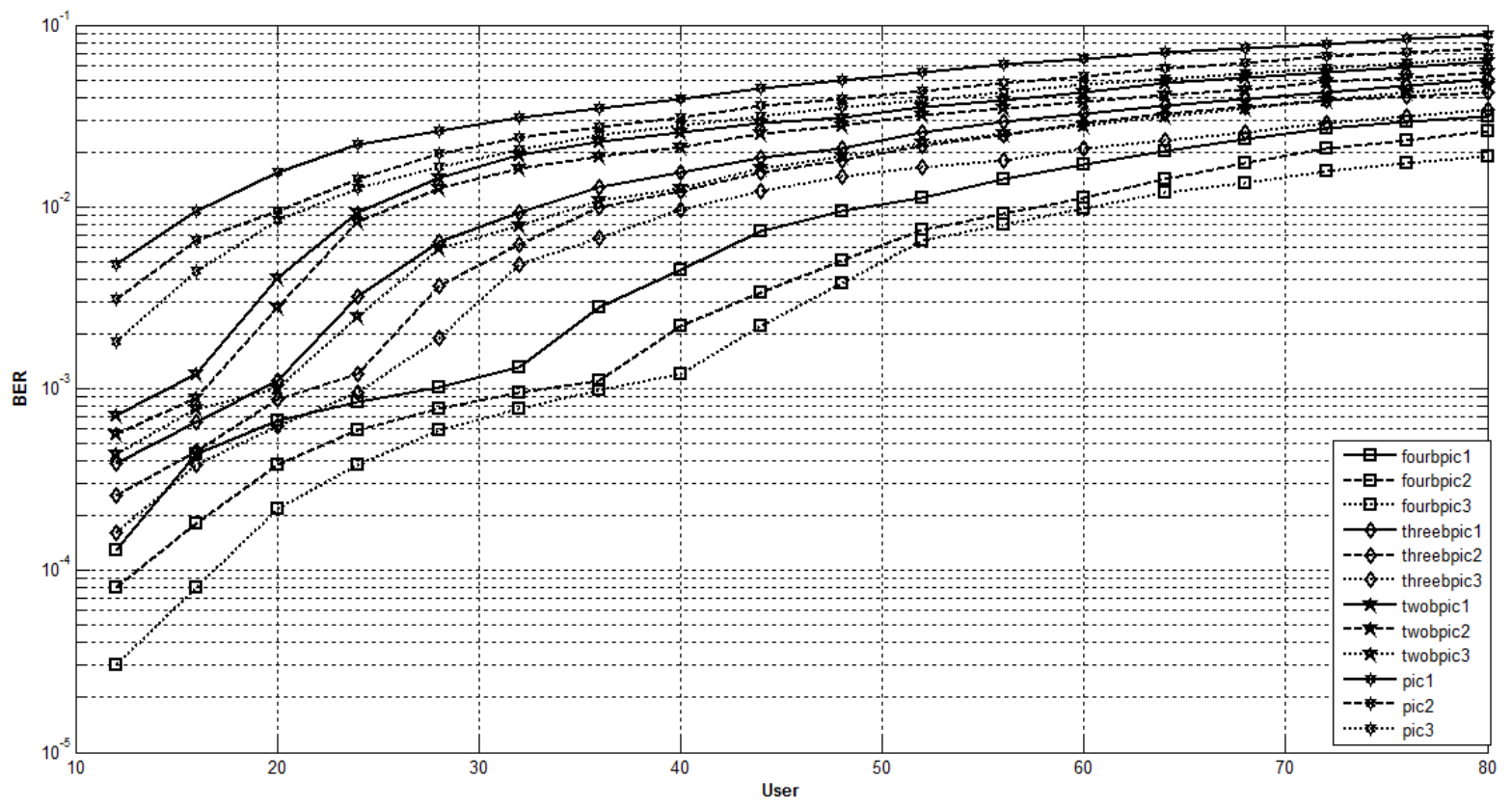

Fig.10 BER performance analysis for power adaptive normal BPIC 


\section{$\triangle 1$ Macrothink}

Network Protocols and Algorithms

ISSN 1943-3581

2012, Vol. 4, No. 1

Fig.10 shows BER performance for the power adaptive (using GA and without NN) but using BPIC. An improvement in BER performance for users 80 is achieved from 0.0885 to 0.0190 at third stage interference cancellation of 4BPIC over singe stage PIC scheme. As expected, with the increase of number of blocks, BER performance improvement is achieved at the cost of greater computation.

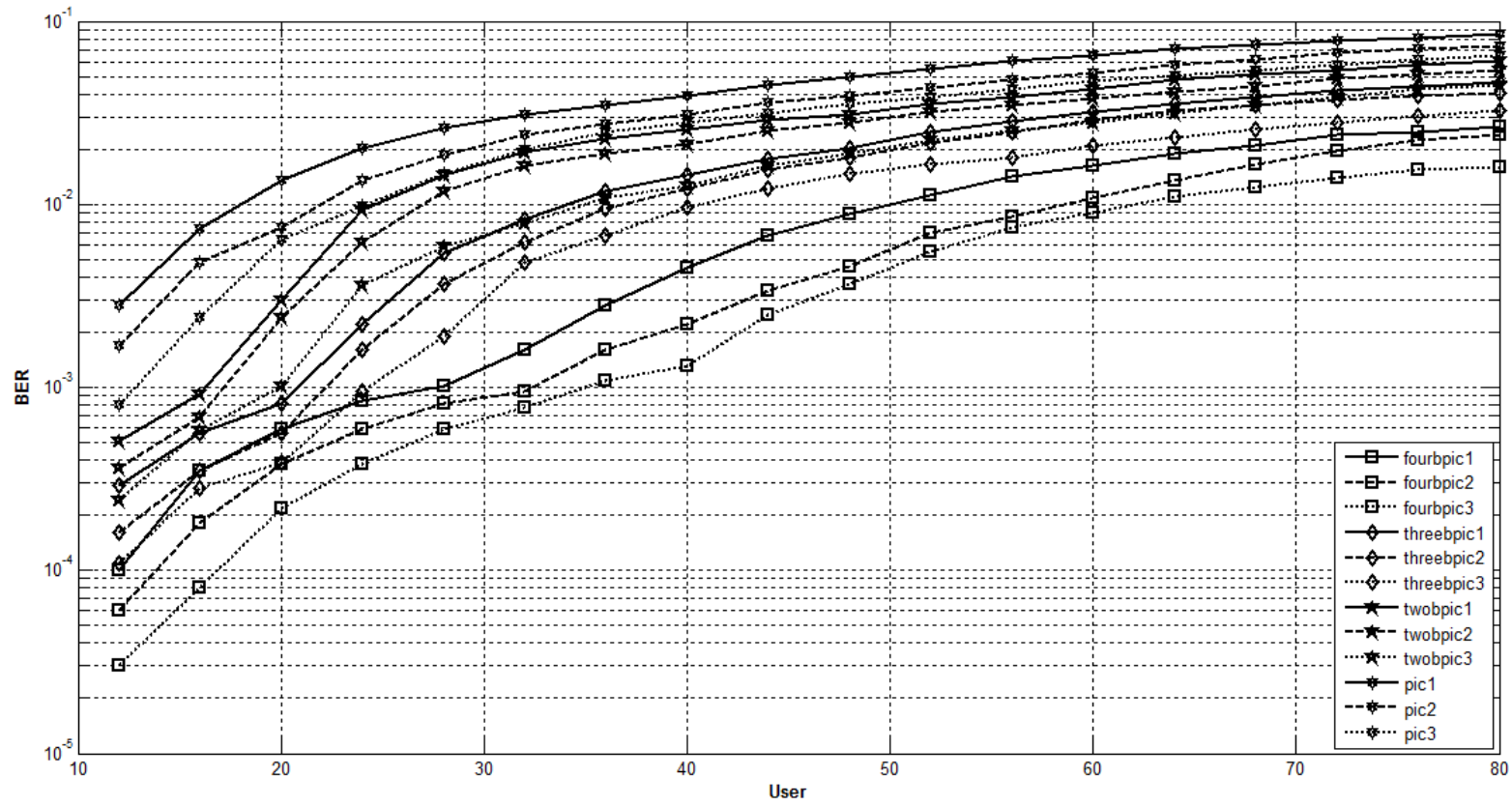

Fig.11 BER performance analysis for non-adaptive NN assisted BPIC.

Fig.11 shows BER performance for the non power adaptive (without GA but using NN) but using BPIC. An improvement in BER performance for users 80 is achieved from 0.0805 to 0.0170 at third stage interference cancellation of 4BPIC over singe stage PIC scheme. Relative performance improvement is achieved for 3BPIC, 2BPIC, 1BPIC etc.

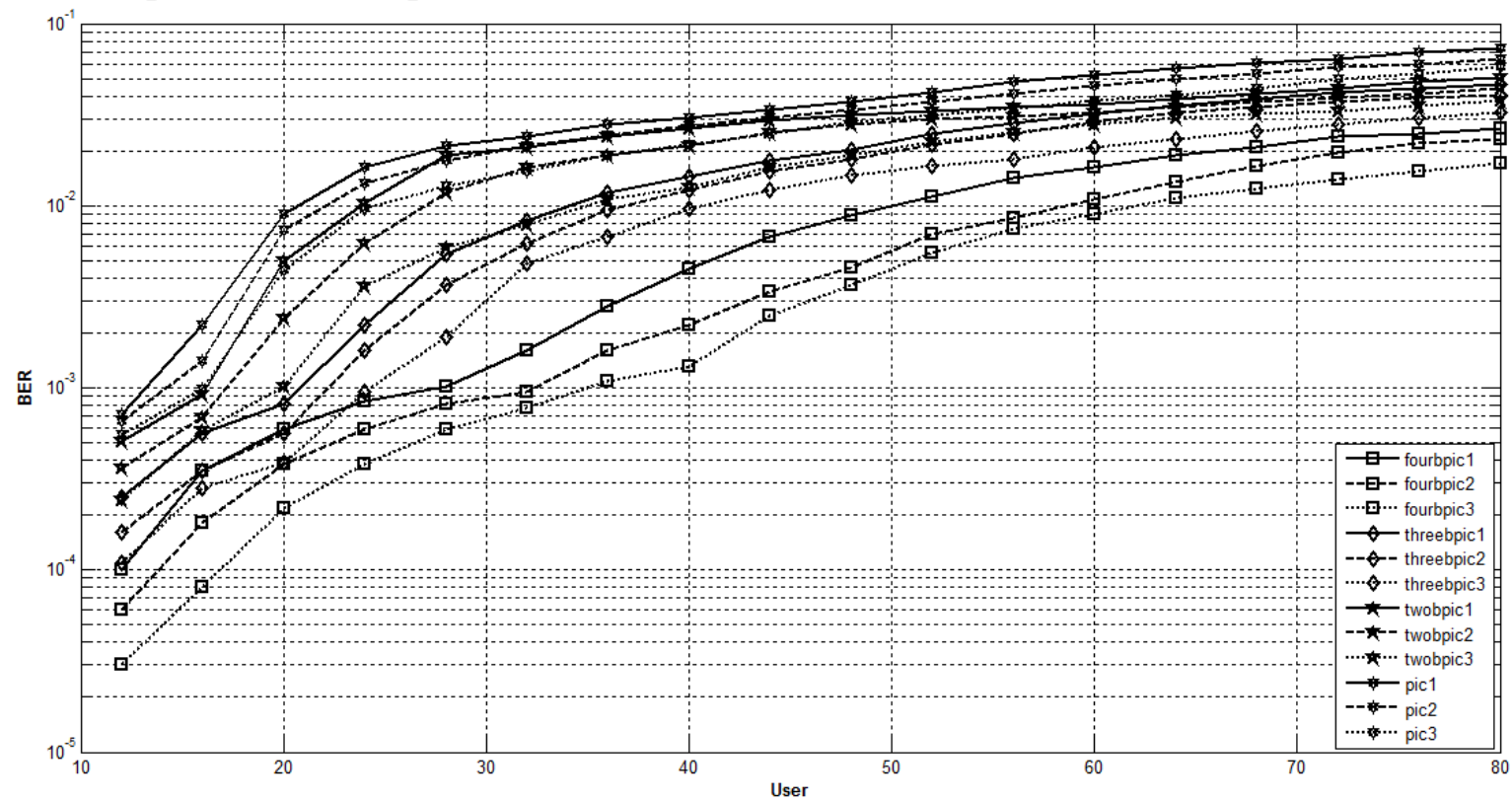

Fig.12 BER performance analysis for adaptive NN assisted BPIC. 
Finally Fig. 12 shows BER performance for the power adaptive (using both GA and NN) and using BPIC.BER performance results are again shown for 4BPIC, 3BPIC, 2BPIC with single, two and three times/stages interference cancelation. An improvement in BER performance for users 80 is achieved from 0.0735 to 0.0155 at third stage interference cancellation of 4BPIC over singe stage PIC scheme.

Numerical values reflect that four block PIC after three stage iterations for power adaptive (using both GA and NN) system can support number of users upto twice the number of subcarriers with BER of the order of 0.0029. It can support users upto three times the number of subcarriers with BER of the order of 0.0115 , while the similar values for non-power adaptive (without both GA and NN) system are $\sim 0.015$ and 0.026 , respectively.

\section{Conclusions and Scope of Future Work}

The paper investigates the scope of usage of GA for adaptive power allocation in CI/MC-CDMA system in order to increase channel capacity, while NN based MMSEC is designed for maintaining BER value under transmit power constraint. Simulation results show that channel capacity and BER performance for the proposed power adaptive system have been improved significantly compared to non-power adaptive system. Simulation results show that NN based four BPIC using proposed power allocation can support number of users upto three times the number of subcarriers at the end of third stage while BER value is of the order of 0.0115. Future work would extend this concept for MC-CDMA based cognitive radio system design for capacity improvement in secondary user under the interference constraint to primary user. Future work would also consider GA based phase optimization of CI codes for reduction in peak to average power ratio (PAPR).

\section{References}

[1] Popovic, B. M.: "Spreading Sequences for Multicarrier CDMA Systems" IEEE Trans. Commun. Vol.47, pp. 918-926(1992); http://dx.doi.org/10.1109/26.771348.

[2] Akansu, A. N. et al: "Wavelet and Subband Transforms: Fundamentals and Communication Applications," IEEE Commun. Mag. pp. 104-115 (1997); http://dx.doi.org/10.1109/35.642839.

[3] Natarajan, B., Nassar, C.R., Shattil, S., Michelini, M., Wu, Z.: "High performance mc-cdma via carrier Interferometry codes", IEEE Trans. on Vehicular Tech. 50, pp.1344-1353, (2001); http://dx.doi.org/10.1109/25.966567.

[4] Maity, S. P., Mukherjee, M.: “On Optimization of CI/MC-CDMA System.” Proc. 20th IEEE Personal, Indoor and Mobile Radio Comm. Symp., Japan, pp. 3203-3207(2009); http://dx.doi.org/10.1109/PIMRC.2009.5450194.

[5] Caire, G., Taricco, G., Biglieri, E.: "Optimum Power Control Over Fading Channels." IEEE Trans. Inform. Theory 45, pp. 1468-1489 (1999); http://dx.doi.org/10.1109/18.771147.

[6] Shen, Z., Andrews, J.G., Evans, B.L.: "Adaptive Resource Allocation in Multi-user 
OFDM Systems with Proportional Rate Constraints." IEEE Trans. on Wireless Commun. 42, pp. 2726-273( 2005); http://dx.doi.org/10.1109/TWC.2005.858010.

[7] Luo, J., Blum, R.S., Cimini, L., Cimini, L., Haimovich, A.: "Power Allocation in a Transmit Diversity System with Mean Channel Gain Information.” IEEE Commun. Lett. 9, pp. 2726-2737 (2005); http://dx.doi.org/10.1109/LCOMM.2005.1461682.

[8] Zhang, J., Matolak, D.W.: "Transmitted Power Allocation/control for Multiband MC-CDMA", Journal of Physical Commun. Vol. 3, pp.139-146 (2009); http://dx.doi.org/10.1016/j.phycom.2009.08.001.

[9] Verdu, S.: "Minimum probability of error for asynchronous Gaussian multiple access channels", IEEE Transactions on Inform. Theory, vol. 32, pp. 85-96(1986); http://dx.doi.org/10.1109/TIT.1986.1057121.

[10] Aazhang, B., Paris, B. P. and Orsak, G. C. : "Neural Networks for Multi-user Detection in Code-Division Multiple-Access Communications", IEEE Transactions on Communications, Vol.40, No.7, pp.1212-1222(1992); http://dx.doi.org/10.1109/26.153366.

[11][11] Kechriotis, G. I. and Manolakos, E. S.: "Hopfield Neural Network Implementation Of the Optimal CDMA Multi-user Detector", IEEE Transactions on Neural Networks, Vol.7, No.1, pp.131-41(1996); http://dx.doi.org/1045-9227(96)00159-2.

[12] Valadon, C. and Tafazolli, R.: "A Fast Adaptive Multiuser Detector For DS-CDMA Communications Based On An Artificial Neural Network Architecture", Proc. Of 5th IEEE International Symposium on Spread Spectrum Techniques and Applications, Vol. 3 , pp. 873 -877(1998); http://dx.doi.org/10.1109/ISSSTA.1998.722503.

[13] Kao, J. W.-H., Berber, S. M. and Kecman, V.: "Blind Multiuser Detector for Chaos-Based CDMA Using Support Vector Machine”, IEEE Transaction on Neural Networks, Vol. 21(2010); http://dx.doi.org/10.1109/TNN.2010.2048758.

[14] Chen, S., Samingan, A. K. and Hanzo, L. "Adaptive Near Minimum Error Rate Training for Neural Networks with Application to Multiuser Detection in CDMA Communication Systems”, Signal Processing, 85(7). pp. 1435-1448 (2005); http://dx.doi.org/10.1016/j.sigpro.2005.02.005.

[15] Matyjas, J. D, Psaromiligkos, I. N, Batalama, S. N and Medley, M. J.: "Fast Converging Minimum Probability of Error Neural Network Receivers for DS-CDMA Communications", IEEE Transactions on Neural Networks, Vol. 15, pp. 445-454 (2004); http://dx.doi.org/10.1117/12.487977.

[16] Liu, S. and Wang, J.: "Blind Adaptive Multiuser Detective Using a Recurrent Neural Network", Proc. In Int. Conf. on Communications, circuits and systems, vol. 2,pp. 1071-1705(2004)

[17] Yee, N., Linnartz, J. P. and Fettweis, G.: "BER of multicarrier CDMA in indoor wireless networks," IEEE Personal Indoor and Mobile Radio Communications (PIMRC) Int. Conference, Sept. 1993, Yokohama, Japan, pp. 109-113.

[18] Thippavajjula, V. and Natarajan, B.: "Parallel Interference Cancellation Techniques for Synchronous Carrier Interferometry/MC-CDMA Uplink," IEEE conf. on Vehicular Technology, pp. 69-78 (2004); http://dx.doi.org/10.1109/VETECF.2004.1400032.

[19] Cai, X. and Akansu, A. N.: "Multicarrier CDMA Systems with Transmit Diversity," IEEE Trans. On52nd Conf. on Vehicular Technology, vol. 6, pp. 2817-2821(2000). 


\section{Macrothink}

[20] Maity, S.P., Hati, S., Maity, S.: "Diversity-Assisted Block PIC for synchronous CI/MC-CDMA uplink system using Genetic Algorithm.” In: Proc. of Third IEEE Int. Conf. on Industrial and Information Systems (ICIIS 2008), pp. 1-6. Indian Institute of Technology, Kharagpur (2008), India; http://dx.doi.org/10.1109/ICIINFS.2008.4798486.

[21] Pratihar, D. K.: Soft Computing, 1st Edition, Narosa Publishing House Pvt. Ltd, 2009.

\section{Copyright Disclaimer}

Copyright reserved by the author(s).

This article is an open-access article distributed under the terms and conditions of the Creative Commons Attribution license (http://creativecommons.org/licenses/by/3.0/). 